\title{
A software tool for auditory and speech perception experimentation
}

\author{
LUIS E. LÓPEZ-BASCUAS \\ Universidad Complutense de Madrid, Madrid, Spain \\ and \\ CARLOS CARRERO MARÍN and FRANCISCO J. SERRADILLA GARCÍA \\ Universidad Politécnica de Madrid, Madrid, Spain
}

\begin{abstract}
A computer program capable of supporting auditory and speech perception experimentation is described. Given a continuum of acoustic stimuli, the program (Paradigm) allows the user to present those stimuli under different, well-known psychophysical paradigms (simple identification, identification with a rating scale, $2 \mathrm{IAX}, \mathrm{ABX}, \mathrm{AXB}$, and oddity task). For discrimination tests, both high uncertainty (roving designs) and minimal psychophysical uncertainty (fixed designs) procedures are available. All the relevant time intervals can be precisely specified, and feedback is also available. Response times can be measured as well. Furthermore, the program stores subjects' responses and provides summaries of experimental results for both individual subjects and groups. The program runs on Microsoft Windows (3.1 or 95) on personal computers equipped with any soundboard.
\end{abstract}

Psychophysical research is usually a painstaking task. The researcher must first design the experiment. This entails the selection of some standard psychophysical paradigm and the setting of its specific variables (e.g., time intervals, ranges, etc.). Second, the stimuli, already organized according to one paradigm, should be presented to the subject. Finally, subjects' responses must be appropriately collected so that the results can be easily inspected by the experimenter and smoothly exported to any statistical package.

The main purpose of this paper is to describe a computer program (Paradigm) developed in our laboratory that undertakes all the tasks mentioned above. The program is intended for psychophysical speech research, although general auditory investigations can also be implemented. Some other programs are available for this type of research. For instance, Read, Buder, and Kent (1992), in their review of seven computer systems for speech research, mention some systems (e.g., Computer Speech Lab, CSL, and Computerized Speech Research Environment, CSRE 4.2) that offer stimulus presentation facilities. Other general purpose programs (e.g., Micro Experimental Laboratory, MEL, and Mathematica) can also be used for stimulus presentation. Although some modules of CSL and CSRE are specifically oriented to help researchers prepare auditory perception experiments,

This work was supported in part by Dirección General de Investigación Científica y Técnica Grants PB93/0363 and PB96/1021. We are grateful to José Manuel lgoa and three anonymous reviewers for helpful comments on earlier versions of this paper. Correspondence concerning this article should be addressed to L. E. López-Bascuas, Departmento de Psicología Básica I, Universidad Complutense de Madrid, Campus de Somosaguas, 28223, Madrid, Spain (e-mail: psbas10@sis.ucm.es). they do not provide the specific routines that Paradigm implements. For example, CSRE offers an experiment control system (ECoS) that consists of two different programs: an experiment generator (ECoSGen) and an experiment controller (ECoSCon). ECoSGen is used to specify the experiment type and set its specific parameters. ECoSGen is more powerful than Paradigm in certain respects-for example, it allows running both deterministic and adaptive experiments, whereas Paradigm is only oriented to deterministic experiments. However, experiment design is easier within the Paradigm environment. The user of Paradigm only has to click on the experiment type he or she wants to run, and Paradigm designs the block formation by itself. In contrast, ECoSGen has a block creation screen where users have to select the order in which the stimuli are presented in each block. This gives more flexibility to the program but makes it more difficult to use. The situation is even more complex for the other systems (i.e., MEL or Mathematica). It can be fairly said that Mathematica basically works as a programming language and, therefore, that users must write their own programs. None of this is true for Paradigm. One of the main advantages of Paradigm is its ease of use, so that even novices can safely work with it.

Paradigm first allows the user to select a given experimental paradigm. In this phase, the experimenter will also choose the stimulus set and fix all the relevant variables for the chosen paradigm. All the time intervals are specified in milliseconds (see below for a discussion of time management). Second, the program controls the presentation of the stimuli through a soundboard that must be available in the computer. Finally, subjects' responses (together with response time measures) can be collected, using the computer keyboard (the experimenter has pre- 
viously selected which keys correspond to which response category). A simple text editor is also implemented so that the researcher can inspect a given set of results. For large sets of data, any other more powerful text editor can be invoked from the application. Paradigm provides summaries for both individual subjects and groups. For a given subject, the presentation of results can be made according to the actual order of presentation in the experiment (which allows the study of sequential effects) or organized by stimulus trials. Thus, three different forms of result presentation are available within Paradigm.

Paradigm assumes that sound files will be stored as wave (WAV) files. WAV files follow the standard format introduced by Microsoft Windows for audio file handling. Because users may have access to different sound file formats, a few words about conversion routines are in order. As Read et al. (1992) point out, there are three main factors that constrain the compatibility of data formats: (1) the environment used by the researcher (Macintosh or IBMcompatible system), (2) the choice between ASCII and binary formats, and (3) the specification of the sound files' headers. Obviously, digitization parameters must be considered too. We have developed software capable of undertaking some conversions. In particular, in Carrero, López-Bascuas, and Serradilla (1996), a program is described that allows interchange between sound files generated by CSRE 4.2. (ADF files) and WAV files, required by Paradigm. The program can be obtained free of charge at the address specified below. For a more detailed description of conversion programs, the reader is referred to Read et al.'s section on the compatibility of data formats. Their Figure 3 is especially telling about using utilities supplied with different packages for data exchanging.

In what follows, we first give some important definitions; then the main paradigm variables are presented. This is followed by a discussion of the different available paradigms themselves and by a section that illustrates the sequence of operations to be followed when using Paradigm. Finally, some important technical aspects of the program are discussed.

\section{Definitions}

Stimulus continuum. A continuum is a set of stimuli whose physical features are all the same, except for one that is changed gradually. Paradigm assumes that the experimenter already has such a continuum.

Paradigms. A paradigm is a schema that defines how the stimuli will be presented. The program implements two identification and four discrimination paradigms.

Trials. A trial is the minimal unit of an experiment. So, after each trial, the subject must give a response. In identification paradigms, one trial equals one stimulus; however, discrimination trials always consist of more than one stimulus.

Blocks. A block is a set of trials. A block must include all the possible types of trials for a given experiment. That is why the minimum length of an experiment is (theoretically) one block. Thus, the length of an experiment is measured in blocks. Blocks allow the experimenter to control the probability of appearance of different trials and the order of appearance of such trials (avoiding having many trials of the same type together in an experiment). Block formation depends on the experimental paradigm, so it will be described later.

Experiments. An experiment is a sequence of blocks. Each block contains the appropriate stimuli, independently randomized.

\section{Paradigm Variables}

There are a number of variables that must be specified for the different experimental paradigms. They are all described here for the sake of clarity.

Time intervals. There are two time intervals: interstimulus intervals (ISIs) and intertrial intervals (ITIs). Both types of intervals can be measured either from the beginning or from the end of the stimuli.

ITIs can be either fixed or variable. When they are fixed, subjects have to give their responses within the specified ITI. In this case, trials always follow a periodic sequence, irrespective of when the subject gave his or her response. On the contrary, under variable ITIs, the time interval between trials will depend on subjects' reaction times, since ITIs will count after the response is given by the subject.

Reaction time. When activated, this variable measures the response time of the subject for each experimental trial. Reaction times are measured from the beginning of the trial until the subject presses any key.

Number of blocks. This variable measures the length of the experiment. Except for 2IAX, where block formation is somewhat more complex (see below), the variable number of blocks determines how many times subjects will be confronted with each type of experimental trial.

Step. Discrimination trials are generated from two stimuli of the continuum. The step variable determines how different the two stimuli will be - that is, how far apart they are within the continuum. Therefore, stimulus $n$ will be paired with the stimulus $n+$ step. The user must be aware that the order of stimuli within a continuum is determined by the order with which the user selected those stimuli when the experiment was designed. Thus stimulus 1 would be the first stimulus selected by the user, stimulus 2 would be the second stimulus selected, and so forth.

Feedback. For discrimination experiments, the user can select the feedback option. Under this option, the program informs the subject about the correctness of his or her answers.

Break. The user can define a break after any block of the experiment.

\section{Experimental Paradigms}

Paradigms will not be described in full detail. The interested reader can consult different references for that 
purpose. For instance, Macmillan and Creelman (1991) provide an introduction to various paradigms in the framework of signal detection theory.

Identification paradigms. Two identification paradigms are available: simple identification and rating scale experiments. These paradigms have been widely employed in the literature (see, e.g., much of the experimental work reviewed in Darwin, 1991). In both cases, each trial is composed of just one stimulus, and each block is formed by all the stimuli in the continuum, appropriately randomized.

In simple identification, the user selects as many response categories as desired. Each category will be attached to one key on the keyboard. Rating scale designs can be implemented in two ways. In one version, subjects first decide to which category the stimulus is more likely to belong and then give a rating (e.g., confidence in their responses). In this case, subjects have to press two keys after each trial, and hence, the experimenter must define two keys for each response category. In the other version, subjects only have to press one key that amalgamates information about the chosen category and the rating given. In this case, only one key for each response category must be defined by the user.

Discrimination paradigms. Discrimination paradigms include $\mathrm{ABX}, \mathrm{AXB}$, Oddity task, and 2IAX. All of them have been employed in auditory and speech research (see, e.g., the material reviewed by Repp, 1984). In order to understand block formation for discrimination paradigms, a new concept must be defined, the concept of $s u b$ block. In discrimination paradigms, two or more stimuli are always presented within a single trial (sometimes one stimulus is repeated twice). A sub-block is made by the different trials that a given pair of acoustic stimuli can generate. For instance, in a 2IAX design, given a pair of stimuli $\mathrm{A}$ and $\mathrm{B}$, a sub-block will be constituted by the four possible trials generated by these two stimuli: AA,
$\mathrm{AB}, \mathrm{BA}$, and $\mathrm{BB}$ (see below, for details on the 2IAX paradigm). The sub-block concept is intended to help the experimenter control the experiment (a given experimental trial appears the same number of times during the experiment, and trials are adequately randomized).

\section{Roving Versus Fixed Designs}

Paradigm implements both fixed and roving discrimination designs. The theoretical implications of using one or the other design have been discussed, for instance, by Kewley-Port, Watson, and Foyle (1988). These authors distinguish between high-uncertainty and minimal psychophysical uncertainty procedures. The former are recommended when the aim of the experiment is to determine how listeners perceive complex stimuli under ordinary conditions, whereas the latter should be employed whenever the underlying sensory capability (resolving power) is the goal of the study. Roving designs favor high-uncertainty conditions, whereas fixed designs place subjects under minimal psychophysical uncertainty testing conditions.

Roving designs. In roving designs, any trial can appear anywhere within an experiment. Therefore, blocks must be formed and randomized in a way that can fulfill this requirement. A roving block is then defined by all the sub-blocks generated by all the possible pairings of stimuli within a continuum (see Figure 1). Then this block is randomized.

Fixed designs. In contrast to roving designs, fixed designs must have all the sub-block trials grouped together within the experiment. To accomplish this, the way in which the blocks are formed and randomized is somewhat changed. In particular, fixed designs will have a fixed number of presentation blocks. This fixed number equals the number of possible pairings of stimuli for a given continuum. Thus, for a 10-stimulus continuum and a one-

\begin{tabular}{|c|c|c|c|c|}
\hline $\begin{array}{l}1 \\
2 \\
1 \\
2\end{array}$ & $\begin{array}{l}2 \\
1 \\
1 \\
2 \\
\end{array}$ & $\begin{array}{l}1 \\
1 \\
2 \\
1 \\
\end{array}$ & $\begin{array}{l}2 \\
3 \\
4 \\
4\end{array}$ & $\begin{array}{l}2 \\
2 \\
3 \\
4\end{array}$ \\
\hline $\begin{array}{l}2 \\
3 \\
2 \\
3 \\
\end{array}$ & $\begin{array}{l}3 \\
2 \\
2 \\
3 \\
\end{array}$ & $\begin{array}{l}3 \\
2 \\
3 \\
2\end{array}$ & $\begin{array}{l}3 \\
1 \\
2 \\
2\end{array}$ & $\begin{array}{l}3 \\
2 \\
3 \\
2\end{array}$ \\
\hline $\begin{array}{l}3 \\
4 \\
3 \\
4 \\
\end{array}$ & $\begin{array}{l}4 \\
3 \\
3 \\
4 \\
\end{array}$ & $\begin{array}{l}4 \\
3 \\
4 \\
3 \\
\end{array}$ & $\begin{array}{l}3 \\
1 \\
2 \\
3\end{array}$ & $\begin{array}{l}3 \\
1 \\
1 \\
4\end{array}$ \\
\hline
\end{tabular}

Block 1

\begin{tabular}{|lll|}
\hline 3 & 3 & 2 \\
2 & 3 & 3 \\
4 & 4 & 3 \\
2 & 2 & 1 \\
1 & 1 & 2 \\
3 & 3 & 4 \\
2 & 2 & 3 \\
1 & 2 & 2 \\
4 & 3 & 3 \\
3 & 2 & 2 \\
3 & 4 & 4 \\
2 & 1 & 1 \\
\hline
\end{tabular}

Block k

Figure 1. Example of a roving discrimination design. The three smaller boxes on the left are sub-blocks for an $\mathrm{ABX}$ design. The bigger boxes are the actual roving blocks that will be presented in the experiment. 
step design, nine presentation blocks will be constructed, since nine are all the possible one-step pairings of those stimuli.

Because all the trials generated by a given pair of stimuli must be grouped together, one sub-block must be repeated a number of times within one presentation block. The number of sub-blocks contained in one presentation block is controlled by the variable number of blocks, in order to be consistent with the definitions given above. Figure 2 shows an example of a fixed discrimination design. It is assumed that there are four stimuli in the continuum. Thus, three presentation blocks are generated. The order of presentation of these blocks and the trials within each block are both randomized.

\section{Oddity Task, ABX, AXB, and 2IAX Paradigms}

In oddity tasks, $\mathrm{ABX}$, and $\mathrm{AXB}$, each trial contains three stimuli. In $\mathrm{ABX}, \mathrm{A}$ and $\mathrm{B}$ are two different stimuli, and $X$ can be either $A$ or $B$. Subjects have to decide whether $\mathrm{X}$ is $\mathrm{A}$ or $\mathrm{B}$. AXB is the same, but now the test stimuli is located between $A$ and $B$. In oddity tasks, three stimuli (one different from the other two) are presented. Subjects have to decide whether the odd stimulus appeared in first, second, or third place. In 2IAX paradigms, only two stimuli are presented to the subjects. They have to decide whether the stimuli are different or the same. Subblocks formed by these four paradigms can be seen in Figure 3.

As was mentioned before, blocks and sub-blocks allow the user to control the experiment carefully. For $\mathrm{ABX}$, $\mathrm{AXB}$, and oddity task, the procedures implemented in Paradigm guarantee that all the possible trials will appear the same number of times throughout the experiment. However, for 2IAX, the situation is more complicated. Notice that, in 2IAX, the same trial can be generated from two different stimuli pairings. Thus, some trials will appear twice as often as other trials (this fact will be reflected in the presentation of the results). The number of times this will occur will depend on the number of stimuli within the continuum and the step selected by the user. Although for most widely used designs (experiments that use small steps) Paradigm generates a well-balanced experiment, users are encouraged to be careful when using 2IAX procedures.

All these paradigms can be used under fixed or roving designs. Figure 4 shows an example of an ABX experiment under a roving design. It assumes a continuum of five stimuli, a two-step design, and a length of three blocks. The specification of these paradigms is made by using the set of variables explained above.
(12)

\begin{tabular}{|ll|}
\hline 1 & 2 \\
2 & 1 \\
1 & 1 \\
2 & 2 \\
\hline
\end{tabular}

(2,3)

\begin{tabular}{|ll|}
\hline 2 & 3 \\
3 & 2 \\
2 & 2 \\
3 & 3 \\
\hline
\end{tabular}

$(3,4)$

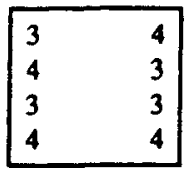

Sub-blocks

Block 1

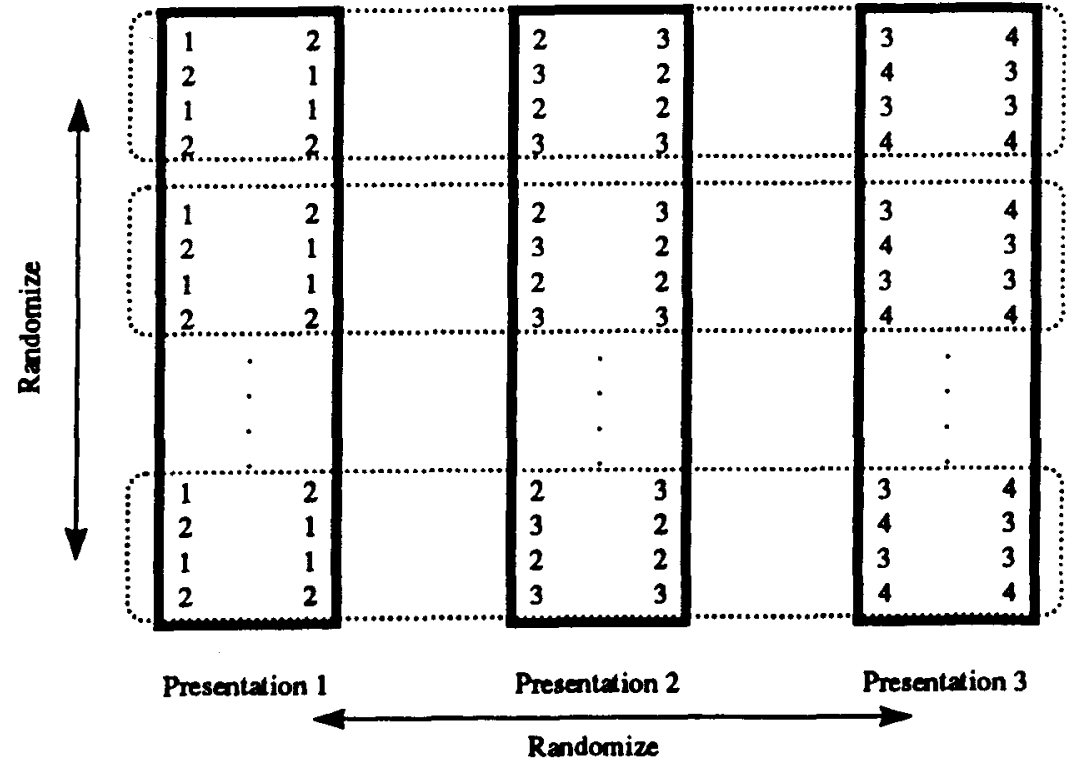

Block 2

Block k

Figure 2. Example of a fixed discrimination design. The three smaller boxes at the top are subblocks for a 2IAX design. The bigger boxes are the three presentation blocks generated by a fourstimulus continuum. See the text for details. 
$\mathrm{ABX}$

\begin{tabular}{|lll|}
\hline 1 & 2 & $1 *$ Response \\
1 & 2 & $2 *$ Response \\
2 & 1 & $1 *$ Response \\
2 & 1 & $2 *$ Response \\
\hline
\end{tabular}

$\mathrm{AXB}$

\begin{tabular}{|lll|}
\hline 1 & 1 & $2 *$ Response \\
1 & 2 & $2 *$ Response \\
2 & 2 & $1 *$ Response \\
2 & 1 & $1 *$ Response \\
\hline
\end{tabular}

\section{Oddity Task}

\begin{tabular}{|lll|}
\hline 1 & 2 & $2 *$ Response \\
2 & 1 & $2 *$ Response \\
2 & 2 & $1 *$ Response \\
2 & 1 & $1 *$ Response \\
1 & 2 & $1 *$ Response \\
1 & 1 & $2 *$ Response \\
\hline
\end{tabular}

2IAX

\begin{tabular}{|ll|}
\hline 1 & $2 *$ Response \\
2 & $1 *$ Response \\
1 & $1 *$ Response \\
2 & $2 *$ Response \\
\hline
\end{tabular}

Figure 3. Sub-blocks for four different discrimination paradigms.

\section{Using Paradigm}

A general block diagram describing the functioning of Paradigm can be inspected in Figure 5. The diagram shows the prescribed order of operations when Paradigm is used.

After running Paradigm, the user may want either to open an already designed experiment or to design a new experiment. If the experiment is already designed, the user may check out the results of a subject or run a new subject. In the latter case, a new subject must be opened, and identification variables must be filled for that subject. After that, the experiment can be run on that subject, and his or her results can be inspected or saved. If the experiment has not been designed yet, the user must first design it. The available paradigms were described earlier, and the relevant variables that define each paradigm have been examined too. So, designing an experiment en- tails the setting of those specific variables. Once the experiment is designed, the user may save the experiment for future use or open a subject again. Then, the experiment can be run, and the results can be saved in a file specified by the user. The user can also see the results for any subject. As was described, there are three possible ways of showing the results of an experiment. In the first one, all the trials, as they appeared in the experiment, are listed together with the responses given by the subject. In the second form, a summary is provided for each subject. In the summary, the number of hits, errors, and omissions can be inspected for each type of trial. Paradigm also provides general summaries for a group of subjects.

When an experiment is run, the screen blanks. Only window buttons are visible on the corners of the screen. However, this will be so if the user maximizes the appli-

$$
\begin{aligned}
& \text { (1,3) } \begin{array}{lll}
1 & 3 & 1 \\
1 & 3 & 3 \\
3 & 1 & 1 \\
3 & 1 & 3
\end{array} \\
& (2,4) \quad \begin{array}{lll}
2 & 4 & 2 \\
2 & 4 & 4 \\
4 & 2 & 2 \\
4 & 2 & 4 \\
\hline
\end{array} \\
& (3,5) \quad \begin{array}{lll}
3 & 5 & 3 \\
3 & 5 & 5 \\
5 & 3 & 3 \\
5 & 3 & 5 \\
\hline
\end{array}
\end{aligned}
$$

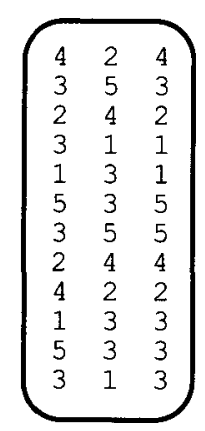

Block 2

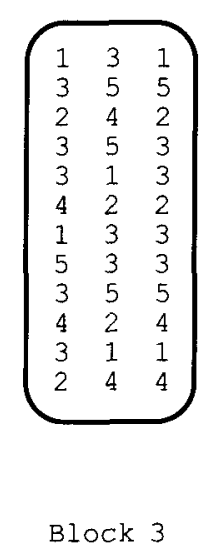

Figure 4. Example of a roving discrimination ABX design. It assumes a continuum of five stimuli, a two-step design, and a length of three blocks. 


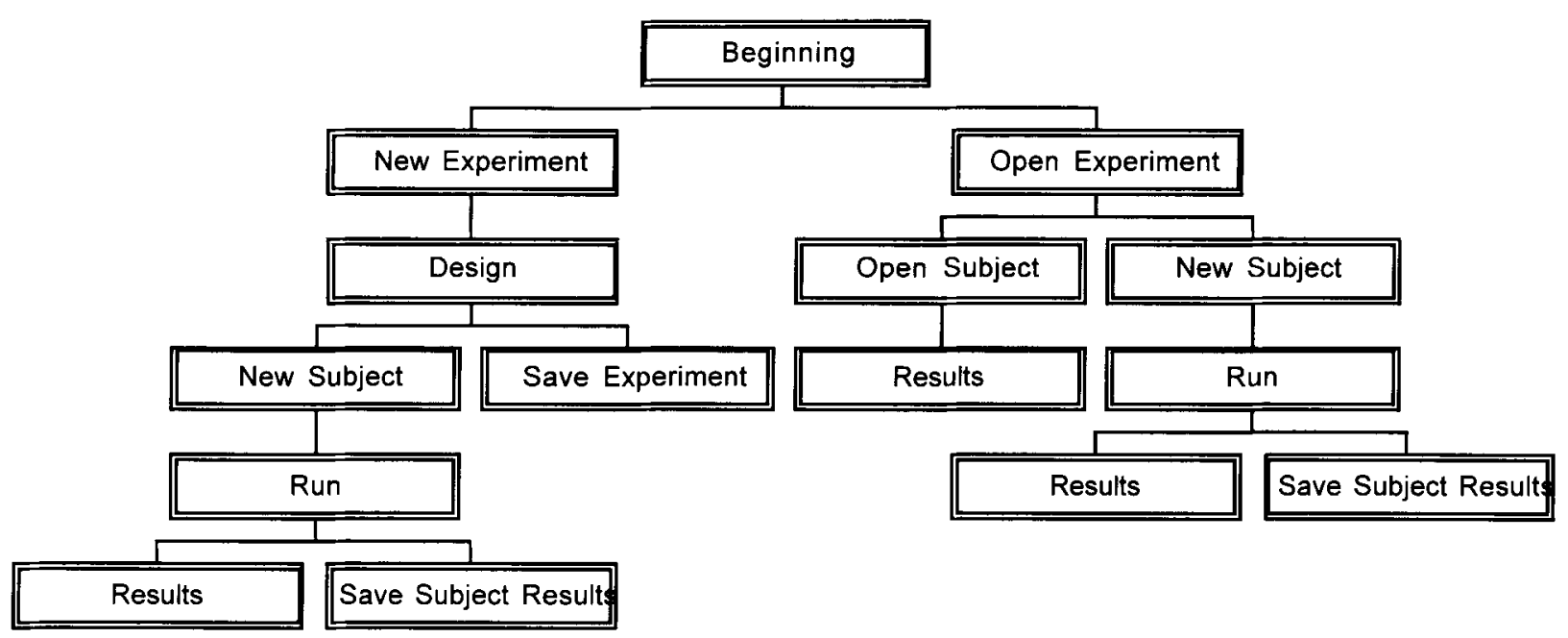

Figure 5. Hierarchy of functions in Paradigm.

cation window. Otherwise, some other icons may remain visible. Thus, we recommend that the application window be maximized whenever an experiment is going to be run.

\section{Technical Aspects}

Time management. Time accuracy is a prerequisite for any auditory or speech perception investigation. Usually a precision of milliseconds is needed. Thus, Paradigm was designed in an effort to meet this requirement. Although the program makes use of the powerful multimedia services included in Windows (mmsystem.dil), new functions and algorithms have been implemented, in order to guarantee sound file management in real time.

Microsoft Windows API (Microsoft Corporation, 1991) provides two different types of audio-managing services: high-level and low-level functions. Although highlevel functions simplify audio management, these functions cannot guarantee our time accuracy requirements. Therefore, Paradigm makes use of low-level functions in order to open or close device drivers, to allocate and prepare audio data blocks, and to write waveform data. Thus, before actually running an experiment, Paradigm goes through the following steps. (1) Audio devices are prepared using the waveOutOpen() function; this function opens an output device and prepares it for sound reproduction. (2) Memory is allocated for audio data blocks; for this purpose, the GlobalAlloc() and GlobalLock() functions are used. (3) Audio data blocks are prepared by using the OutPrepareHeader () function. Our new algorithms implement all these steps in the proper sequence. All these new algorithms have been carefully tested. According to our tests, accuracy of presentation is always within the $-0.5-+0.5 \mathrm{msec}$ range around the specified time interval. After completing this process, sound playback can be initiated. Paradigm uses the waveOutWrite() function for sending audio data blocks to output devices.
Response time measurements employ the timeGetTime() function extensively. By subtracting two values provided by this function, it is possible to measure time intervals accurately. Figure 6 shows an example of the algorithm used for presenting a given stimulus and collecting subjects' responses under a fixed ITI. As can be seen, once the stimulus is presented, using the waveOutWrite () function, the program remains in a loop, waiting for the response of the subject. The loop finishes after the completion of the specified ITI. Within the loop, the program stores the first response provided by the subject, using the PeekMessage function. Our time estimations (using the recommended equipment, see below) indicate that the PeekMessage function is executed one time each $0.4 \mathrm{msec}$. Therefore, Paradigm guarantees accurate time measurements in milliseconds.

Nevertheless, speed of sound file management is also dependent on the actual equipment (computer and soundboard) being used. Using the recommended equipment (see below), ITIs must be at least $160 \mathrm{msec}$, to allow proper functioning of the application. Since subjects must give the response during this time, this restriction is never a real limitation. Better equipments can even reduce this minimum ITI. We have noticed that minimum ITIs may vary considerably from one environment to another. To avoid ill-functioning of the application whenever specified ITIs are not long enough, an error message will appear on the screen, asking the user to change interval times.

\section{Design and Implementation}

Paradigm was developed with Borland $\mathrm{C}++$ on Windows 3.1. as a programming tool. The ObjectWindows class library simplifies windows programing and, therefore, has been extensively used. Hence, the application follows an Object Oriented Programming (OOP) approach, combined with processes of event-based pro- 


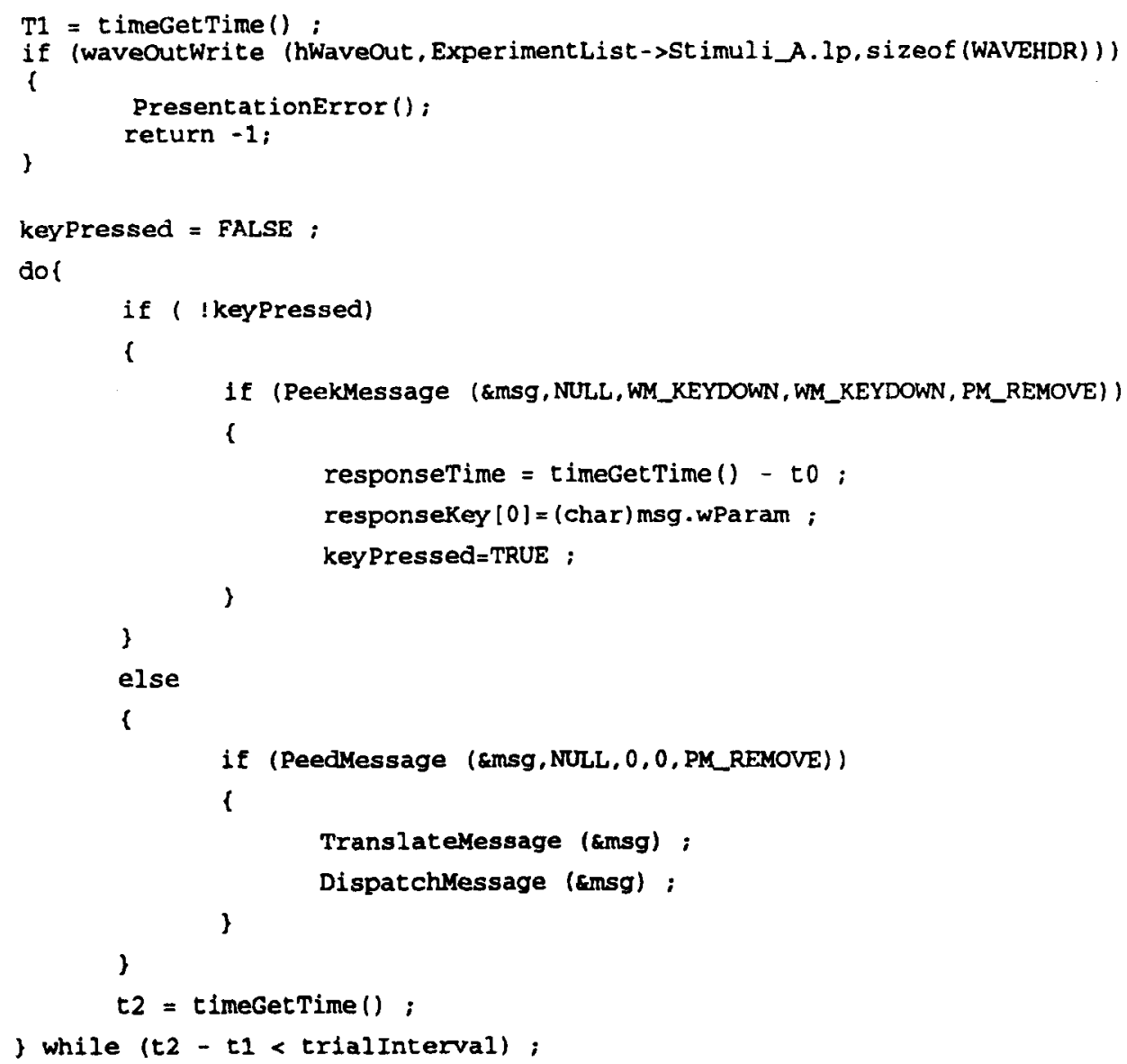

Figure 6. Algorithm for presenting the last stimulus of a given trial and for collecting the response of the subject under a fixed intertrial interval.

gramming. The design of the application is structured in classes, treating the experimental paradigms as objects. This way, all the advantages of the OOP approach can be used and will permit, for instance, adding new experimental paradigms in the future with minimal effort.

\section{Hardware Requirements}

The minimum recommended equipment for a good functioning of this tool is a PC computer with an Intel 486/ 33-MHz microprocessor and 8-Mb RAM. A soundboard is also required, in order to handle the WAV sound files. In all our tests of time accuracy, a Creative Labs Soundblaster ( 16 bits) with DSP has been employed. Computers running in a network environment are not recommended, since other computing tasks might affect time management within Paradigm. The program runs on Microsoft Windows 3.1 or Windows 95.

\section{Availability}

Paradigm and Convert programs can be obtained free of charge at the following address: http://www.sia.evi. upm.es/grvpos/paradigm.html

\section{REFERENCES}

Carrero, C., López-Bascuas, L. E., \& Serradilla, F. (1996). Un programa para la traducción de formatos en ficheros de sonido [A computer program for converting formats of sound files]. Psicológica, 17, 173-180.

DARWIN, C. J. (1991). The relationship between speech perception and the perception of other sounds. In I. G. Mattingly \& M. StuddertKennedy (Eds.), Modularity and the motor theory of speech perception (pp. 239-259). Hillsdale, NJ: Erlbaum.

Kewley-Port, D., Watson, C. S., \& Foyle, D. C. (1988). Auditory temporal acuity in relation to category boundaries: Speech and nonspeech stimuli. Journal of the Acoustical Society of America, 83, 1133-1145.

Macmillan, N. A., \& Creelman, C. D. (1991). Detection theory: A user's guide. Cambridge: Cambridge University Press.

Microsoft CORPORATION (1991). Microsoft Windows multimedia programmer's workbook. Redmond, WA: Microsoft Press.

READ, C., BudER, E. H., \& KENT, R. D. (1992). Speech analysis systems: An evaluation. Journal of Speech \& Hearing Research, 35, 314-332.

REPP, B. H. (1984). Categorical perception: Issues, methods, findings. In N. J. Lass (Ed.), Speech and language: Advances in basic research and practice (Vol, 10, pp. 243-335). New York: Academic Press.

(Manuscript received March 5, 1997; revision accepted for publication April 2, 1998.) 